\title{
Triality of conformal extensions of three kinds of special relativity
}

\author{
Han-Ying Guo \\ CCAST (World Laboratory), P.O. Box 8730, Beijing 100080, China, and \\ Institute of Theoretical Physics, Chinese Academy of Sciences, Beijing 100080, China \\ Bin Zhou \\ Department of Physics, Beijing Normal University, Beijing 100875, China \\ Yu Tian \\ Department of Physics, Beijing Institute of Technology, Beijing 100081, China \\ Zhan Xu \\ Physics Department, Tsinghua University, Beijing 100084, China
}

(Dated: February 8, 2007)

\begin{abstract}
The conformal extensions of three kinds of special relativity with $I S O(1,3) / S O(1,4) / S O(2,3)$ invariance on Mink/dS/AdS space, respectively, are realized on an $S O(2,4) / \mathbb{Z}_{2}$ invariant projective null cone $[\mathcal{N}]$ as the (projective) boundary of the 5-d AdS space. The relations among the conformal Mink/dS/AdS spaces, the motions of light signals and the conformal field theories on them can be given. Thus, there should be a triality for these conformal issues and the conjectured AdS/CFT correspondence.
\end{abstract}

PACS numbers: 11.25.Hf, 03.30.+p, 95.30.Sf

\section{INTRODUCTION}

'It is well known that in Einstein's special relativity on the Minkowski(Mink) space $M_{1,3}$ with the Mink lineClement $d s_{M}^{2}$, massless particles and light signals move in inertia along null geodesics, satisfying $d s_{M}^{2}=0$, which are invariant under conformal transformations. Thus, the symmetry of their motions should be enlarged from the Poincaré group $I S O(1,3)$ to the conformal group with 15 Qpạ́rameters.

'. It has been shown that on an almost equal footing with Einstein's special relativity, there are two other kinds of the de Sitter (dS)/anti-dS (AdS)-invariant special relativity on the dS/AdS space of radius $R$ with Beltrami coordiJhate atlas, denoted as the BdS/BAdS space, with the Beltrami line-element $d s_{B \pm}^{2}$, respectively [1-8]. In the dS/AdS special relativity, massless particles and light signals move also in inertia along null straight world lines with constant coordinate velocity components and satisfying $d s_{B \pm}^{2}=0$. These properties are also invariant under conformal transformations, respectively. Thus, the symmetry of these motions should also be enlarged from the dS/AdS-group $S O(1,4) / S O(2,3)$ to the conformal group, respectively.

Under the conformal transformations, the Mink/dS/AdS space is not closed, often with some events sent to infinity and vice versa, respectively. Thus, each of these spaces must be extended so that they are closed under the conformal transformations. It is also known that dS and AdS are conformally flat. In the viewpoint of the conformal triality, however, they are also conformally dS/AdS, respectively. In other words, locally dS, AdS and Mink spaces are conformally equivalent. However, globally it is not the case, due to different topologies of these spaces.
In this paper, we show that in addition to the conformal extension of Einstein's special relativity on $M_{1,3}$, the conformal extension of the dS/AdS special relativity on 4-d $\mathrm{dS} /$ AdS space can also be realized on the same null cone $\mathcal{N}$ modulo the projective equivalence " $\sim$ " in a $(2+4)$-d Mink space $M_{2,4}$, respectively. In order to be distinguished from $\mathcal{N}$, the resulted 4 -d space as a quotient space is denoted by $[\mathcal{N}]:=\mathcal{N} / \sim$. Actually, the conformal transformations on each space are induced by the $S O(2,4)$ isometries on $M_{2,4}$ and these spaces are mapped to each other by certain Weyl conformal mappings. Thus, the projective null cone $[\mathcal{N}]$ and the physics on it are the conformal extension and compactification of $M_{1,3} / \mathrm{dS}_{4} / \mathrm{AdS}_{4}$, underlying the conformal extension of Poincaré/dS/AdS-invariant special relativity, respectively. And the conformal transformations on $M_{1,3}$, $\mathrm{dS}_{4}$ and $\mathrm{AdS}_{4}$ can also be equivalently related. Thus, the conformal physics, such as the motions of light signals and the conformal field theories (CFTs), can be mapped from one space to another. Here, by the term CFT, we mainly refer to theories that are invariant under the 15-parameter conformal transformation groups. So, there should be a triality of these conformal issues.

Since the projective boundary of a 5-d AdS space, $\partial\left(\mathrm{AdS}_{5}\right)$, is just $[\mathcal{N}]$, the conformal $\mathrm{dS}_{4}$ and $\mathrm{AdS}_{4}$ can also be included in $\partial\left(\mathrm{AdS}_{5}\right)$, similar to the conformal $M_{1,3}$. Thus, if the AdS/CFT correspondence [9] is conjectured, there should be three versions of AdS/CFT correspondence [10].

This paper is arranged as follows. In Sec. II, we very briefly introduce why there are three kinds of special relativity and show why their conformal extensions on the conformal Mink/dS/AdS spaces are eventually on the same $[\mathcal{N}]$. In Sec. III, we study the physics on these conformal 
extensions and their relations. We first show the triality of these conformal extensions via Weyl mappings for any two of them in III A. Then we focus on the motion of free massless particles along the null geodesics in III B. We also consider the relations among the CFTs on them and the triad for the AdS/CFT-correspondence in III C. Finally, we end with a few remarks.

\section{THREE KINDS OF SPECIAL RELATIVITY AND THEIR CONFORMAL EXTENSIONS}

In this section, we first very briefly introduce why there are two other kinds of special relativity with dS/AdS invariance, respectively, in addition to Einstein's special relativity on the Mink space. Then we show why their conformal extensions on the conformal Mink/dS/AdS spaces are eventually on the same null-cone modulo projective equivalence in a $(2+4)$-d Mink space.

\section{A. Three kinds of special relativity}

It has been shown that, based on the principle of relativity and the postulate on invariant universal constants, the $\mathrm{dS} / \mathrm{AdS}$ special relativity on the dS/AdS space with Beltrami coordinate atlas can be set up [1-8]. Thus, there are three kinds of special relativity on an almost equal footing. The principle is the same as that in Einstein's special relativity and the postulate requires that in addition to the speed of light $c$ there is another invariant universal constant $R$ as the curvature radius of the $\mathrm{dS} / \mathrm{AdS}$ space, respectively.

The dS/AdS space with radius $R$ can be viewed as a 4 -d hyperboloid $H_{\theta}(\theta= \pm 1)$ embedded in $M_{1,4} / M_{2,3}$, respectively:

$$
\begin{aligned}
& H_{\theta}: \eta_{\mu \nu} \xi^{\mu} \xi^{\nu}-\theta\left(\xi^{4}\right)^{2}=\eta_{\theta A B} \xi^{A} \xi^{B}=-\theta R^{2} \lessgtr 0 \\
& d s_{H_{\theta}}^{2}=\eta_{\mu \nu} d \xi^{\mu} d \xi^{\nu}-\theta\left(d \xi^{4}\right)^{2}=\eta_{\theta A B} d \xi^{A} d \xi^{B}
\end{aligned}
$$

where $\mu, \nu=0, \cdots, 3 ; A, B=0, \cdots, 4$. Let us consider a kind of uniform "great circular" motions for a particle with mass $m_{R}$, defined by a conserved 5-d angular momentum on $H_{\theta} \subset M_{1,4} / M_{2,3}$ :

$$
\frac{d \mathcal{L}^{A B}}{d s_{H_{\theta}}}=0, \quad \mathcal{L}^{A B}:=m_{R}\left(\xi^{A} \frac{d \xi^{B}}{d s_{H_{\theta}}}-\xi^{B} \frac{d \xi^{A}}{d s_{H_{\theta}}}\right) .
$$

There is an Einstein-like formula for the particle

$$
-\frac{1}{2 R^{2}} \mathcal{L}^{A B} \mathcal{L}_{A B}=m_{R}^{2}, \quad \mathcal{L}_{A B}=\eta_{\theta A C} \eta_{\theta B D} \mathcal{L}^{C D}
$$

For a massless particle or a light signal with $m_{R}=0$, similar uniform "circular" motion can also be defined so long as the proper-time $d s_{H_{\theta}}$ is replaced by an affine parameter $\lambda_{\theta}$ and there is no $m_{R}$ in the counterpart of $\mathcal{L}^{A B}$ in (2.3), respectively. Namely,

$$
\frac{d L^{A B}}{d \lambda_{\theta}}=0, \quad L^{A B}:=\xi^{A} K^{B}-\xi^{B} K^{A}, \quad K^{A}:=\frac{d \xi^{A}}{d \lambda_{\theta}} .
$$

There is also an Einstein-like formula for the particle

$$
-\frac{1}{2 R^{2}} L^{A B} L_{A B}=0, \quad L_{A B}=\eta_{\theta A C} \eta_{\theta B D} L^{C D} .
$$

These properties are symmetrically transformed under the linear transformations of the $\mathrm{dS} / \mathrm{AdS}$-group, respectively.

The Beltrami coordinate atlas without antipodal identification on $d S_{4} / A d S_{4}$ can be defined patch by patch $[3,4]$. In the patch $\xi^{4}>0$ in $H_{+}$, say, the Beltrami coordinates are:

$$
x_{+}^{\mu}=R \xi^{\mu} / \xi^{4}, \quad \xi^{4}>0 .
$$

In this patch, there are a condition from (2.1) and a Beltrami metric from (2.2) [1-4] as follows

$$
\begin{aligned}
& \sigma\left(x_{+}\right):=1-R^{-2} \eta_{\mu \nu} x_{+}^{\mu} x_{+}^{\nu}>0, \\
& d s_{B+}^{2}=\left[\frac{\eta_{\mu \nu}}{\sigma\left(x_{+}\right)}+\frac{\eta_{\mu \sigma} \eta_{\nu \rho} x_{+}^{\sigma} x_{+}^{\rho}}{R^{2} \sigma\left(x_{+}\right)^{2}}\right] d x_{+}^{\mu} d x_{+}^{\nu} .
\end{aligned}
$$

They are invariant under the fractional linear transformations with a common denominator, denoted as the FLTs of the dS-group

$$
\begin{aligned}
x^{\mu} \rightarrow \tilde{x}^{\mu} & = \pm \sigma^{1 / 2}(a) \sigma^{-1}(a, x)\left(x^{\nu}-a^{\nu}\right) D_{\nu}^{\mu}, \\
D_{\nu}^{\mu} & =L_{\nu}^{\mu}+R^{-2} \eta_{\nu \lambda} a^{\lambda} a^{\kappa}\left(\sigma(a)+\sigma^{1 / 2}(a)\right)^{-1} L_{\kappa}^{\mu}, \\
L & :=\left(L_{\nu}^{\mu}\right) \in S O(1,3),
\end{aligned}
$$

which transform a point $A\left(a^{\mu}\right), \sigma\left(a^{\mu}\right)>0$ in the Beltramisystem $S(x)$ to the origin in the system $\tilde{S}(\tilde{x})$.

It can be proved that all geodesics of the Beltrami-metric (2.9) are indeed straight world lines. Actually, along a timelike or a null geodesic a massive particle or a light signal moves with constant coordinate velocity components, respectively. For the particle with mass $m_{R}$ there is a set of conserved observables:

$$
\begin{array}{cl}
p^{\mu}=m_{R} \sigma^{-1}(x) \frac{d x^{\mu}}{d s}, & \frac{d p^{\mu}}{d s}=0 ; \\
l^{\mu \nu}=x^{\mu} p^{\nu}-x^{\nu} p^{\mu}, & \frac{d l^{\mu \nu}}{d s}=0 .
\end{array}
$$

These are the pseudo 4-momentum, pseudo 4-angularmomentum of the particle, which constitute the conserved 5 -d angular momentum in (2.3). The second equation in (2.11) is just the equation for the timelike geodesic of the Beltrami metric (2.9), from which it follows that the second equation in (2.12) is satisfied and its coordinate velocity components are constants:

$$
\frac{d x^{i}}{d t}=v^{i}=\text { const. }, \quad i=1,2,3 .
$$


For the case of a massless particle or a light signal, it is also the case for its motion along the null geodesic. Namely, there is a set of conserved observables:

$$
\begin{array}{cl}
k^{\mu}=\sigma^{-1}(x) \frac{d x^{\mu}}{d \lambda_{+}}, & \frac{d k^{\mu}}{d \lambda_{+}}=0 ; \\
l^{\mu \nu}=x^{\mu} k^{\nu}-x^{\nu} k^{\mu}, & \frac{d l^{\mu \nu}}{d \lambda_{+}}=0 .
\end{array}
$$

These are the pseudo 4-momentum, pseudo 4-angularmomentum of the massless particle, which constitute the conserved 5-d angular momentum in (2.5).

In terms of $p^{\mu}$ and $l^{\mu \nu}$, the Einstein-like formula (2.4) becomes:

$$
\begin{gathered}
E^{2}-p^{2}-\frac{1}{2 R^{2}} l_{(1,3)}^{2}=m_{R}^{2} \\
E^{2}=m_{R}^{2} c^{4}+p^{2} c^{2}+\frac{c^{2}}{R^{2}} j^{2}-\frac{c^{4}}{R^{2}} b^{2},
\end{gathered}
$$

with energy $E$, momentum $p^{i}, p_{i}=\delta_{i j} p^{j}$, "boost" $b^{i}, b_{i}=$ $\delta_{i j} b^{j}$ and 3 -angular momentum $j^{i}, j_{i}=\delta_{i j} j^{j}$. For the massless case, the formula is the same with $m_{R}=0$.

Thus, there is indeed a law of inertia invariant under the FLTs (2.10) in the Beltrami atlas patch by patch for particles and light signals. And the principle and the postulate hold and the dS-invariant special relativity can further be set up. Further, the simultaneity, light-cone, horizon and other issues can be well formulated in the Beltrami atlas patch by patch.

It is important that for light signals, $d s_{B+}^{2}=0$. This leads to the conformal extension of the BdS space invariant under conformal transformation group with 15 parameters, in analogy with that of the Mink space.

Similar approach can be applied to the case of AdS space with $\theta=-1$.

It is also clear that under the limit $R \rightarrow \infty$, all these issues go back to the corresponding ones in Einstein's special relativity on the Mink space.

\section{B. The conformal extensions on the null cone}

The conformal extensions of BdS/BAdS space can be realized first via the hyperboloid $H_{ \pm}$by introducing a scaling variable $\kappa \neq 0$ and a set of coordinates $\zeta^{\hat{A}}$, respectively,

$$
\begin{array}{ll}
\zeta^{\mu}:=\kappa \xi^{\mu}, \zeta^{4}:=\kappa \xi^{4}, \zeta^{5}:=\kappa R, & \text { for } H_{+} ; \\
\zeta^{\mu}:=\kappa \xi^{\mu}, \zeta^{4}:=\kappa R, \zeta^{5}:=\kappa \xi^{4}, & \text { for } H_{-},
\end{array}
$$

and secondly back to the Beltrami atlas. Under such a scaling, eq. (2.1) turns out to be a null cone

$$
\mathcal{N}: \eta_{\hat{A} \hat{B}} \zeta^{\hat{A}} \zeta^{\hat{B}}=0, \quad \eta_{\hat{A} \hat{B}}=\operatorname{diag}(J,-1,1),
$$

where $J=\operatorname{diag}(1,-1,-1,-1), \Xi:=\left(\zeta^{\hat{A}}\right)=\left(\zeta, \zeta^{4}, \zeta^{5}\right) \neq 0$. Now $H_{ \pm}$are parts of $\mathcal{N} \subset M_{2,4}$. The Beltrami -coordinates $x_{ \pm}^{\mu}$ can be obtained from coordinates $\zeta^{\hat{A}}$, too.
The null cone $\mathcal{N}(2.20)$ is $S O(2,4)$-invariant in $M_{2,4}$ and there is the projective equivalence relation " $\sim$ " on $M_{2,4}-$ $\{0\}: \Xi^{\prime} \sim \Xi$ if and only if there is a number $c \neq 0$ satisfying $\zeta^{\prime \hat{A}}=c \zeta^{\hat{A}}$. The resulted quotient space $[\mathcal{N}]:=\mathcal{N} / \sim$ is a 4-d submanifold of $\mathbb{R} P^{5}$, homeomorphic to $S^{1} \times S^{3}$. Intuitively, an equivalence class of $\Xi \in \mathcal{N}$ can be viewed as the null straight line passing through both $\Xi$ and the origin of $M_{2,4}$. The origin is not included in the equivalence class, however. In this sense $[\mathcal{N}]$ consists of all the null straight lines through the origin. Thus, an $S O(2,4)$ transformation on $M_{2,4}$ induces a transformation on $[\mathcal{N}]$, sending one null straight line to another.

Thus, the dS/AdS-hyperboloid $H_{\theta}$ can be embedded into the same $\mathcal{N}$. When the metric on $M_{2,4}$ is pulled back to $\mathcal{N}$, it is conformal to $d s_{H_{\theta}}^{2}$ :

$$
d \chi_{\mathcal{N}}^{2}:=\eta_{\hat{A} \hat{B}} d \zeta_{\mathcal{N}}^{\hat{A}} d \zeta_{\mathcal{N}}^{\hat{B}}=\kappa^{2} d s_{H_{\theta}}^{2}
$$

In terms of the inhomogeneous projective coordinates or the Beltrami coordinates, we have

$$
d \chi_{\mathcal{N}}^{2}=\kappa^{2} d s_{B \theta}^{2} .
$$

Consequentially, $S O(2,4)$ transformations on (2.20) induce conformal transformations on $H_{\theta}$, respectively:

$$
d s_{H_{\theta}}^{2}=\rho^{2} d s_{H_{\theta}}^{2}, \quad \rho=\frac{\kappa}{\kappa^{\prime}}= \begin{cases}\zeta^{5} / \zeta^{\prime 5}, & \text { for } H_{+} ; \\ \zeta^{4} / \zeta^{\prime 4}, & \text { for } H_{-} .\end{cases}
$$

Or

$$
d s_{B \theta}^{\prime 2}=\rho^{2} d s_{B \theta}^{2}, \quad \rho=\frac{\kappa}{\kappa^{\prime}}
$$

According to eqs. (2.18) and (2.19), $H_{ \pm}$can be viewed as the intersection of $\mathcal{N}$ and the hyperplanes $P_{+}: \zeta^{5}=R$ and $P_{-}: \zeta^{4}=R$, respectively. Since $H_{\theta}$ is only part of $\mathcal{N}$, with $\zeta^{5}$ (for $H_{+}$) or $\zeta^{4}$ (for $H_{-}$) nonzero, it is quite possible for an $S O(2,4)$ transformation to send a point in $H_{\theta}$, with nonzero $\zeta^{5}$ or $\zeta^{4}$, to another one with zero $\zeta^{5}$ or $\zeta^{4}$, and vice versa. Thus, $H_{\theta}$ are, in fact, not closed under the induced conformal transformations. To be closed, $H_{\theta}$ must be extended into the whole $[\mathcal{N}]$. Thus, $[\mathcal{N}]$ is the conformal extension of both dS and AdS spaces.

It is clear that back to the Beltrami atlas, say (2.7) for the BdS, as inhomogeneous projective coordinates, the conformal BdS/BAdS-metric follows, respectively. Thus, the conformal extension of the dS/AdS-invariant special relativity can be set up for massless particles and light signals, respectively.

As is well known, the conformal Mink space can also be obtained from the the same null cone (see, e.g. [11]). To this end, we introduce a set of new coordinates

$$
\zeta^{ \pm}:=\left(\zeta^{5} \pm \zeta^{4}\right) / \sqrt{2}
$$

and assign coordinates, with $R$ the same as before,

$$
x^{\mu}:=R \zeta^{\mu} / \zeta^{-}, \quad x^{+}:=R \zeta^{+} / \zeta^{-}
$$


to those points with $\zeta^{-} \neq 0$. Then eq. (2.20) becomes $x^{+}=-\eta_{\mu \nu} x^{\mu} x^{\nu} /(2 R)$, and the metric (2.21) becomes

$$
d \chi_{\mathcal{N}}^{2}=\left(\zeta^{-} / R\right)^{2} d s_{M}^{2}, \quad d s_{M}^{2}:=\eta_{\mu \nu} d x^{\mu} d x^{\nu}
$$

And, a $S O(2,4)$ transformation induces a conformal transformation on the Mink space:

$$
d s_{M}^{2} \rightarrow d s_{M}^{\prime 2}=\rho^{2} d s_{M}^{2}, \quad \rho=\zeta^{-} / \zeta^{\prime-} .
$$

Similarly, the Mink space $M_{1,3}$ can be regarded as the intersection of $\mathcal{N}$ and the hyperplane $P_{M}: \zeta^{-}=R$ by identifying $\left(x^{\mu}\right)$ with $\left(x^{\mu},\left(x^{+}-R\right) / \sqrt{2},\left(x^{+}+R\right) / \sqrt{2}\right) \in \mathcal{N}$. The Mink space is not closed for these conformal transformations, too. Thus, the Mink space needs to be extended, resulting in the space $[\mathcal{N}] \cong S^{1} \times S^{3}$.

\section{THE TRIALITY OF NULL PHYSICS ON CONFORMAL MINK, DS AND ADS SPACES}

Let us now study the null physics on the conformal Mink/dS/AdS spaces and their relations via Weyl mappings. We also explain why there should be a triality of the conjectured AdS/CFT-correspondence.

\section{A. The triality of conformal Mink, dS and AdS spaces}

According to the above discussion, Mink and $H_{\theta}$ spaces and their conformal extensions with the same $R$ can be related by Weyl conformal mappings as follows. A point in $d S$, say, is first viewed as a point in $P_{+} \cap \mathcal{N}$. Then a point in $P_{-} \cap \mathcal{N}$ equivalent to it could be found, in general. However, it is possible that a point in one space could not be mapped into another space, or could not find an inverse image in another space. We solve it elsewhere. Thus the mapping from the conformal extension of $d S_{4}$ to that of $A d S_{4}$ is established.

For example, a point $\xi_{+}:=\left(\xi_{+}^{0}, \ldots, \xi_{+}^{4}\right) \in H_{+}$can be mapped to a point in $H_{-}$with the following Beltrami coordinates:

$$
x_{-}^{\mu}:=R \zeta^{\mu} / \zeta^{5}=\xi_{+}^{\mu} .
$$

As another example, the Weyl conformal mapping sending a point with coordinates $\left(x^{\mu}\right)$ in the Mink space to a point in the BdS space with coordinates $\left(x_{+}^{\mu}\right)$ reads

$$
x_{+}^{\mu}=-\sqrt{2} x^{\mu}\left(1+\frac{1}{2 R^{2}} \eta_{\rho \sigma} x^{\rho} x^{\sigma}\right)^{-1} .
$$

This is just the conformally flat coordinate transformation for the BdS-metric (2.9), which is also known as a stereographic projection with an inverse transformation

$$
x^{\mu}=-\sqrt{2} x_{+}^{\mu}\left(1 \mp \sqrt{\sigma\left(x_{+}\right)}\right)^{-1} .
$$

The sign $\mp$ is opposite to the $\operatorname{sign}$ of $\xi^{4} \gtrless 0$ in the BdS space.

It is important that the normal vectors of $P_{+}, P_{-}$and $P_{M}$ are timelike, spacelike and null, respectively, and that $P_{+} \cap \mathcal{N}, P_{-} \cap \mathcal{N}$ and $P_{M} \cap \mathcal{N}$ are dS, AdS and Mink space, respectively. This can be generalized: given a hyperplane off the origin, its intersection with $\mathcal{N}$ is dS, AdS or Mink if its normal vector is timelike, spacelike or null, respectively.

We have shown that Mink/dS/AdS spaces can all be conformally extended to the same $[\mathcal{N}]$, so that they can be conformally mapped from one to another. A conformal transformation on one space is, in fact, also a conformal transformation on another space. And all these conformal transformations are induced from the $S O(2,4)$ transformations, forming a group $S O(2,4) / \mathbb{Z}_{2}$, due to the equivalence relation on $\mathcal{N}$. Therefore, from the viewpoint of conformal transformations, these three kinds of spaces and the CFTs on them are just same. We refer to this fact as the triality of conformal extensions of these spaces.

\section{B. Motion of free massless particles: Null geodesics}

As was mentioned earlier, similar to a massive particle a free massless test particle or a light signal in the dS space has the conserved 5-d angular momentum (2.5). Namely,

$$
L^{A B}:=\xi^{A} K^{B}-\xi^{B} K^{A}, \quad K^{A}:=\frac{d \xi^{A}}{d \lambda}, \quad \frac{d L^{A B}}{d \lambda}=0,
$$

where $\lambda=\lambda_{+}$is an affine parameter. The geometric meaning of the Beltrami coordinates and the fact that geodesics have equations of straight lines [3, 4] imply that a geodesic is the intersection of $\Sigma$ and dS-hyperboloid $H_{+}$in (2.1), where $\Sigma$ is some 2 -d plane passing through the origin of the 5 -d Minkowski space $M_{1,4}$. It can be proved that, when the geodesic is null, it is in fact a straight line in $M_{1,4}$, having the equation $\xi^{A}=\xi_{0}^{A}+\lambda v^{A}$ for some constants $\xi_{0}^{A}$ and $v^{A}$, satisfying $\eta_{A B} \xi_{0}^{A} v^{B}=\eta_{A B} v^{A} v^{B}=0$. Thus the 5-d momentum $K^{A}=v^{A}$ of the null geodesic is also conserved:

$$
\frac{d K^{A}}{d \lambda}=0
$$

Using the relations (2.18), we can obtain

$$
L^{A B}=\frac{1}{\kappa^{2}} \frac{d \psi}{d \lambda} \mathcal{L}^{A B}, \quad P^{A}=\frac{1}{\kappa^{2} R^{2}} \frac{d \psi}{d \lambda} \mathcal{L}^{5 A},
$$

where $\psi=\psi(\lambda)$ is certain a parameter and the 6 - $\mathrm{d}$ angular momentum $\mathcal{L}^{\hat{A} \hat{B}}$ is defined as

$$
\mathcal{L}^{\hat{A} \hat{B}}:=\zeta^{\hat{A}} \frac{d \zeta^{\hat{B}}}{d \psi}-\zeta^{\hat{B}} \frac{d \zeta^{\hat{A}}}{d \psi} .
$$

It is conserved if

$$
d \psi=\kappa^{2} d \lambda
$$


For a massless particle in the AdS space, there are similar issues.

In the Mink-case, the 4-d momentum $k_{M}^{\mu}$ and the angular momentum $l_{M}^{\mu \nu}$ are conserved for a light signal,

$$
k_{M}^{\mu}:=\frac{d x^{\mu}}{d \lambda}, \quad l_{M}^{\mu \nu}:=x^{\mu} k_{M}^{\nu}-x^{\nu} k_{M}^{\mu} .
$$

Similarly, the 6-d angular momentum defined by (3.7) can be related to $k_{M}^{\mu}$ and $l_{M}^{\mu \nu}$ by

$$
\begin{aligned}
& \mathcal{L}^{\mu \nu}=\frac{d \lambda}{d \psi} \kappa^{2} l_{M}^{\mu \nu} \quad \mathcal{L}^{4 \nu}=\frac{1}{\sqrt{2}}\left(\mathcal{L}^{+\nu}-\mathcal{L}^{-\nu}\right), \\
& \mathcal{L}^{5 \nu}=\frac{1}{\sqrt{2}}\left(\mathcal{L}^{+\nu}+\mathcal{L}^{-\nu}\right), \quad \mathcal{L}^{45}=\mathcal{L}^{+-}
\end{aligned}
$$

where

$$
\begin{aligned}
\mathcal{L}^{-\nu} & =\frac{d \lambda}{d \psi} \kappa^{2} R k_{M}^{\nu}, \\
\mathcal{L}^{+\nu} & =\frac{d \lambda}{d \psi} \kappa^{2}\left(x^{+} k_{M}^{\nu}-x^{\nu} \frac{d x^{+}}{d \lambda}\right), \\
\mathcal{L}^{+-} & =-\frac{d \lambda}{d \psi} \kappa^{2} R \frac{d x^{+}}{d \lambda} .
\end{aligned}
$$

If eq. (3.8) is satisfied, then the above 6-d angular momentum is also conserved.

For a massless free particle, its equation of motion in $[\mathcal{N}]$ is not unique in terms of $\zeta^{\hat{A}}$, because $\zeta^{\hat{A}}=\zeta^{\hat{A}}(\psi)$ and $\zeta^{\hat{A}}=\zeta^{\prime \hat{A}}\left(\psi^{\prime}\right):=\rho\left(\psi^{\prime}\right) \zeta^{\hat{A}}\left(\psi\left(\psi^{\prime}\right)\right)$ are equivalent, with $\psi=$ $\psi\left(\psi^{\prime}\right)$ a reparametrization. Formally, there are the angular momenta $\mathcal{L}^{\hat{A} \hat{B}}$ and $\mathcal{L}^{\prime \hat{A} \hat{B}}\left(\psi^{\prime}\right)$ for the same particle. But, a reparametrization can always be chosen so that $\mathcal{L}^{\prime \hat{A} \hat{B}}\left(\psi^{\prime}\right)$ is still conserved.

Consequently, the world line is lying in a 2 -d plane $\Sigma$ passing through the origin of $M_{2,4}$, which is also contained in $\mathcal{N} \subset M_{2,4}$ except for the origin. Thus, the world line $\Sigma-$ $\{0\} / \sim$ is a projective straight line in $[\mathcal{N}]$ : in the Beltrami coordinate on $\mathrm{dS}$ or AdS, or in the Mink coordinate, its equations look like

$$
x^{\mu}(s)=x_{0}^{\mu}+s c^{\mu},
$$

where $x_{0}^{\mu}$ and $c^{\mu}$ are some constants while $s$ is the curve parameter. Hence, the world line is a null geodesic $[3,4]$. The relation of its 5 -d angular momentum and $\mathcal{L}^{\hat{A} \hat{B}}$ is as shown in eqs. (3.6), etc. This coincides with the well-known fact that null geodesics are conformally invariant up to a reparametrization.

Thus, under the Weyl mapping (3.2) from $M_{1,3}$ to $\mathrm{dS}_{4}$, null geodesics in the Mink space $M_{1,3}$ is mapped to null geodesics in $\mathrm{dS}_{4}$. What's more, for all these geodesics, no matter in $M_{1,3}$ or $\mathrm{dS}_{4}$, their equations in the Minkowski coordinates or Beltrami coordinates are all in the form of (3.13). As indicated in section II A, all geodesics in dS or AdS spaces have the similar form of equations in any Beltrami coordinate system. Hence it is acceptable that Beltrami coordinates in dS or AdS spaces play the role of Minkowski coordinates in SR. That is, Beltrami coordinates are inertial coordinates.

\section{On CFT and AdS/CFT correspondence}

Let us now consider other conformal issues on the conformal Mink/dS/AdS spaces and the relations among them.

The generators of the conformal group on the Mink space are

$$
\begin{aligned}
& \hat{p}_{\mu}:=\partial_{\mu}, \quad \hat{l}_{\mu \nu}:=x_{\mu} \partial_{\nu}-x_{\nu} \partial_{\mu}, \\
& \hat{D}:=x^{\mu} \partial_{\mu}, \quad \hat{s}_{\mu}:=-x \cdot x \partial_{\mu}+2 x_{\mu} x^{\nu} \partial_{\nu} .
\end{aligned}
$$

A CFT in the Mink space must be invariant under the action of these generators. The coordinates $x^{\mu}$ can be extended to be a set of coordinates $\left(x^{\mu}, \kappa, \phi\right)$ on $M_{2,4}-\left\{\zeta^{-}=\right.$ $0\}$, where $\kappa$ is the scaling factor introduced before

$$
\kappa=\frac{\zeta^{-}}{R}, \quad \phi:=\eta_{\hat{A} \hat{B}} \zeta^{\hat{A}} \zeta^{\hat{B}} .
$$

Thus the Mink space is described by $\kappa=1$ and $\phi=0$. Then it can be verified that

$$
\begin{aligned}
& \hat{p}_{\mu}=\frac{1}{R} \hat{\mathcal{L}}_{+\mu}, \quad \hat{l}_{\mu \nu}=\hat{\mathcal{L}}_{\mu \nu}, \\
& \hat{D}=\hat{\mathcal{D}}+\hat{\mathcal{L}}_{-+}, \quad \hat{s}_{\mu}=2 x_{\mu} \hat{\mathcal{D}}+2 R \hat{\mathcal{L}}_{-\mu},
\end{aligned}
$$

where

$$
\hat{\mathcal{D}}:=\zeta^{\hat{A}} \frac{\partial}{\partial \zeta^{\hat{A}}}
$$

is the generator of scaling in $M_{2,4}$, while

$$
\hat{\mathcal{L}}_{\hat{A} \hat{B}}:=\zeta_{\hat{A}} \frac{\partial}{\partial \zeta^{\hat{B}}}-\zeta_{\hat{B}} \frac{\partial}{\partial \zeta^{\hat{A}}}
$$

are generators of $\mathfrak{s o}(2,4)$. Since $\hat{\mathcal{D}}$ is commutative with each $\hat{\mathcal{L}}_{\hat{A} \hat{B}}$, it does not matter that the conformal generators of the Mink space differ from those of $M_{2,4}$ by a vector field along $\hat{\mathcal{D}}$ [see, eqs. (3.18)]. This coincides with (i) the idea that the equivalence relation $\sim$ will be considered on $\mathcal{N}$, and (ii) the fact the conformal transformations in the Mink space is induced from, but not the same as, the $S O(2,4)$-transformations on $\mathcal{N}$. In fact, a quantity in the Mink space can be realized by homogeneous function of degree zero in $M_{2,4}-\{0\}$. In this way $\mathcal{D}$ somehow could be dropped directly.

Generators of conformal transformations on dS/AdS spaces, or specially on BdS/BAdS spaces, can also be given as the ones of $\mathfrak{s o}(2,4)$. Thus, they can be related by the Weyl conformal mappings such as (3.1) and (3.2). Correspondingly, the CFTs in these spaces are also related by these mappings. Since the Maxwell equations are the simplest CFT, as an illustration, we show how the sourceless Maxwell equations

$$
d \boldsymbol{F}=0, \quad * d * \boldsymbol{F}=0,
$$

where $*$ is the Hodge dual operator, are related among them. 
Consider the Weyl conformal mapping $\psi: M_{1,3} \rightarrow \mathrm{dS}_{4}$ as shown in eq. (3.2):

$$
\psi^{*} \boldsymbol{g}=\Omega^{2} \boldsymbol{\eta}, \quad \Omega=\sqrt{2}\left(1-\frac{1}{2 R^{2}} \eta_{\mu \nu} x^{\mu} x^{\nu}\right)^{-1},
$$

with $\boldsymbol{g}$ the metric (2.9) of $\mathrm{BdS}_{4}, \boldsymbol{\eta}$ the one in (2.27). If $\boldsymbol{F}_{\mathrm{dS}}$ is the Maxwell field in $\mathrm{dS}_{4}$, its equations follow

$$
d \boldsymbol{F}_{\mathrm{dS}}=0, \quad \star d \star \boldsymbol{F}_{\mathrm{dS}}=0,
$$

where $\star$ is the dual operator with respect to $\boldsymbol{g}$. We pull $\boldsymbol{F}_{\mathrm{dS}}$ back to the Mink space, resulting in

$$
\boldsymbol{F}=\psi^{*} \boldsymbol{F}_{\mathrm{dS}} .
$$

Thus $d \boldsymbol{F}=d\left(\psi^{*} \boldsymbol{F}_{\mathrm{dS}}\right)=\psi^{*} d \boldsymbol{F}_{\mathrm{dS}}=0$ is satisfied. It can be verified that

$$
\psi^{*}\left(\star d \star \boldsymbol{F}_{\mathrm{dS}}\right)=\Omega^{-2}[* d * \boldsymbol{F}] .
$$

Therefore, in the Mink space, $\boldsymbol{F}$ as in eq. (3.24) is a sourceless electromagnetic field: eqs. (3.21) are satisfied. In this way the Weyl conformal mapping $\psi: M_{1,3} \rightarrow \mathrm{BdS}_{4}$ relates a sourceless electromagnetic field $\boldsymbol{F}_{\mathrm{dS}}$ in the BdS space to a sourceless $\boldsymbol{F}$ in the Mink space.

Similarly, the approach can be applied to other CFTs between dS and AdS spaces, AdS and Mink spaces and so on. Basically, the CFTs in Mink/dS/AdS spaces, in which all the relevant fields are assumed to behave well as the infinity points are approached, can be unified together. The former is merely a realization of the latter.

For the AdS/CFT correspondence, there should also be a triality.

The $\mathrm{AdS}_{5}$ can be embedded into $M_{2,4}$ as a hypersurface $\mathcal{S}:$

$$
\mathcal{S}: \eta_{\hat{A} \hat{B}} \zeta^{\hat{A}} \zeta^{\hat{B}}=R_{5}^{2}, \quad \text { with } R_{5}>0 .
$$

If antipodal points in $\mathcal{S}$ are identified, the resulted space, denoted by $\mathcal{S} / \mathbb{Z}_{2}$, is still homeomorphic to $\mathcal{S} \cong \mathrm{AdS}_{5}$. In the projective space $\mathbb{R} P^{5}=M_{2,4}-\{0\} / \sim$, the quotient space of those $\zeta^{\hat{A}}$ satisfying $\eta_{\hat{A} \hat{B}} \zeta^{\hat{A}} \zeta^{\hat{B}}>0$ are homeomorphic to $\mathcal{S} / \mathbb{Z}_{2} \cong \mathrm{AdS}_{5}$. Identifying $\mathrm{AdS}_{5}$ with this quotient space, then

$$
\partial\left(\operatorname{AdS}_{5}\right)=[\mathcal{N}]
$$

Thus, due to the triality of the CFTs in conformal Mink/dS/AdS spaces, there should be three AdS/CFT correspondences starting from the well-known AdS/CFT correspondence [9]. Namely, there should be the AdS/CFT correspondence between $A d S_{5}$ and the $\mathrm{dS}_{4} / \mathrm{AdS}_{4}$, respectively, in addition to that between $\mathrm{AdS}_{5}$ and the Mink space. Clearly, this triplet of the AdS/CFT correspondence can be generalized to any dimensions whenever the AdS/CFT correspondence is conjectured.

\section{CONCLUDING REMARKS}

It is emphasized that since there are three kinds of special relativity with $I S O(1,3) / S O(1,4) / S O(2,3)$ invariance, respectively, there should be the triality of their conformal extensions. Actually, similar to the inertia feature of the Mink coordinates on the Mink space $M_{1,3}$, the Beltrami systems on the $\mathrm{dS} / \mathrm{AdS}$ space $\mathrm{dS}_{4} / \mathrm{AdS}_{4}$ play the same role of inertia, respectively. In each of three kinds of special relativity, massive particles or massless particles move uniformly along the timelike or null geodesics with constant coordinate velocity components, respectively. Thus, their motions are of inertia. In fact, as was mentioned, three kinds of special relativity with the Poincaré/dS/AdS-invariance can first be set up based on the principle of relativity and the postulate on the invariant universal constant(s). While for massless particles and light signals, the symmetry should be enlarged from the Poincaré/dS/AdS-group to the conformal group $S O(2,4) / \mathbb{Z}_{2}$, respectively. On the other hand, we may also set up these realizations with the conformal group invariance, basing on the principle of relativity with respect to massless particles and light signals alone, in principle. And for the massive cases, the theory with conformal group symmetry should break back to the special relativity of Poincaré/dS/AdS invariance, respectively.

We have shown that the conformal extensions of three kinds of special relativity can be realized on $[\mathcal{N}]$, the projective null cone modulo projective equivalence, as the 4d $S O(2,4) / \mathbb{Z}_{2}$ conformal Mink/dS/AdS spaces with the same constant $R$. In general, each space may have its own constant $R$ while our approach works similarly. The Weyl conformal mappings among these spaces, the motions of light signals and the CFTs on them have been given. Thus, there should be a triality for these conformal issues. Since $\partial\left(\mathrm{AdS}_{5}\right)=[\mathcal{N}]$, there should be a triplet of the conjectured AdS/CFT correspondence.

It should also be noted that the supersymmetry extension of the $\mathfrak{s o}(2,4)$ can also be viewed as that of dS/AdSalgebras as subalgebras in bosonic sector, in addition to the conventional approach with Poincaré algebra as a subalgebra.

\section{Acknowledgments}

We would like to thank Z. Chang, C.-G. Huang, W.L. Huang, Q.K. Lu, X.C. Song, S.K. Wang, K. Wu, X.N. $\mathrm{Wu}$ and C.J. Zhu for valuable discussions. C.G. Huang attended partly and made contributions to this work. This work is partly supported by NSFC under Grant Nos. 10375087, 90503002, 10347148, 10547002, 10505004 and 10605005 . 
[1] The original idea is due to K.H. Look (Q.K. Lu), "Why the Minkowski Metric Must Be Used?" 1970 (unpublished).

[2] K.H. Look, C.L. Tsou (Z.L. Zou) and H.Y. Kuo (H.-Y. Guo), Acta Physica Sinica 23 (1974) 225; Nature (Shanghai, Suppl.), Modern Physics 1 (1980) 97; H.Y. Kuo, Kexue Tongbao (Chinese Science Bulletin) 22 (1977) 487 (all in Chinese); H.Y. Kuo, Proceedings of the 2nd Marcel Grossmann Meeting on General Relativity, ed. by R. Ruffini, (North-Holland Pub. 1982) 801; H.Y. Guo, Nucl. Phys. B (Proc. Suppl.) 6 (1989) 381.

[3] H.-Y. Guo, C.-G. Huang, Z. Xu and B. Zhou, Mod. Phys. Lett. A19 (2004) 1701; hep-th/0311156.

[4] H.-Y. Guo, C.-G. Huang, Z. Xu and B. Zhou, Phys. Lett. A331 (2004) 1; hep-th/0403171.

[5] H.-Y. Guo, C.-G. Huang, and B. Zhou, Europhys. Lett. 72 [6] (2005) 1045; hep-th/0404010.

[6] H.-Y. Guo, C.-G. Huang, Z. Xu and B. Zhou, Chinese Phys.
Lett. 22 (2005) 2477; hep-th/0508094.

[7] C.-G. Huang, H.-Y. Guo, Y. Tian, Z. Xu and B. Zhou, hep-th/0403013; Y. Tian, H.-Y. Guo, C.-G. Huang, Xu Z, Zhou B, Phys. Rev. D71 (2005) 044030.

[8] Q. K. Lu, Commun. Theor. Phys. 44 (2005) 389.

[9] J. Maldacena, Adv. Theor. Math. Phys. 2 (1998) 231; Int. J. Theor. Phys. 381113 (1999); hep-th/9711200.

[10] B. Zhou and H.-Y. Guo, Conformal triality of de Sitter, Minkowski and anti-de Sitter spaces, in Differential Geometry and Physics, Proc. of 23rd ICDGMTP, Tianjin, Aug. 20-25, 2005. Ed. M.L. Ge and W. Zhang, World Scien. 2006, 503-512; hep-th/0512235.

[11] R. Penrose and W. Rindler, Spinors and Space-Time: Volume 2, Spinor and Twistor Methods in Space-Time Geometry, Cambridge University Press, 1988. 